\title{
Captodative Substitution: A Strategy for Enhancing the Conductivity of Molecular Electronic Devices
}

\author{
Thijs Stuyver, ${ }^{1}$ Tao Zeng, ${ }^{2}$ Yuta Tsuji, ${ }^{3}$ Stijn Fias, ${ }^{1}$ Paul Geerlings, ${ }^{1}$ Frank De Proft ${ }^{*}$ \\ ${ }^{1}$ Algemene Chemie, Vrije Universiteit Brussel, Pleinlaan 2, 1050 Brussels, Belgium (Member \\ of the QCMM Ghent-Brussels Alliance Group) \\ ${ }^{2}$ Department of Chemistry, Carleton University, Ottawa, Ontario K1S5B6, Canada \\ ${ }^{3}$ Education Center for Global Leaders in Molecular Systems for Devices, Kyushu \\ University, Nishi-ku, Fukuoka 819-0395, Japan
}

\begin{abstract}
We explore a new strategy to tune the conductivity of molecular electronic devices: captodative substitution. We demonstrate that a careful design of such substitution schemes on a benzene parental structure can enhance the conductivity by almost an order of magnitude under small bias. Once this new strategy has been established, we apply it to molecular wires and demonstrate that it enables the unprecedented anti-Ohmic design of wires whose conductivity increases with the length. Overall, the captodative substitution approach provides a very promising pathway towards full chemical control of the conductivity of molecules
\end{abstract}

\footnotetext{
* To whom correspondence should be addressed. E-mail: fdeprof@vub.ac.be
} 
which opens up the possibility to design molecular switches with an improved on/off ratio among others.

\section{Introduction}

The effect of substitution on the transport properties of conjugated molecules has received significant attention lately. Most studies up to this point have focused on their influence on the occurrence of Quantum Interference (QI) ${ }^{\dagger}$ around the Fermi level. Yoshizawa and co-workers demonstrated that heteroatom substitution generally only shifts QI features away or towards the Fermi level, but does not eliminate it completely. $\$$ However, the experimental and computational results of Lissau et al. seem to suggest that carefully designed donor-acceptor substitution schemes in combination with environmental influences such as an applied electric field are capable of such a feat. ${ }^{\S}$ The gradual suppression of the QI upon substitution with more pronounced push-pull substituents observed in their work was rationalized through Valence Bond (VB) arguments: QI occurs as long as cross-conjugated resonance structures

† Solomon, G. C.; Andrews, D. Q.; Hansen, T.; Goldsmith, R. H.; Wasielewski, M. R.; Van Duyne, R. P.; Ratner, M. A. Understanding Quantum Interference in Coherent Molecular Conduction. J. Chem. Phys. 2008, 129, 054701.

$\$$ Li, X.; Staykov, A.; Yoshizawa, K. Orbital Views of the Electron Transport through Heterocyclic Aromatic Hydrocarbons. Theor. Chem. Acc. 2011, 130, 765-774.

$\S$ Lissau, H.; Frisenda, R.; Olsen, S. T.; Jevric, M.; Parker, C. R.; Kadziola, A.;

Hansen, T.; van der Zant, H. S. J.; Nielsen, M. B.; Mikkelsen, K. V. Tracking

Molecular Resonance Forms of Donor-Acceptor Push-Pull Molecules by Single-

Molecule Conductance Experiments. Nat. Commun. 2015, 6, 10233. 
dominate the resonance hybrid; it is diminished when the push-pull mechanism raises the percentage of a non-cross-conjugated resonance structure. According to this reasoning, the weight of the cross-conjugating resonance structure could be probed by examining the singlemolecule conductance. Tsuji et al. recently derived theoretically that the introduction of heteroatoms in the linker units connecting the molecule to the contacts does not influence QI in the region in the transmission spectrum limited by the HOMO-LUMO peaks (cf. Linker Theorem). ${ }^{* *}$ Garner and co-workers focused on the influence of electron-withdrawing and electron-donating substituents on the position of QI features in the region between the peaks corresponding to HOMO and LUMO in the transmission spectrum for aromatic molecules. ${ }^{\dagger \dagger}$

The direct enhancement of conductance under small bias without the exploitation of interference effects has received much less attention until recently. Some (mainly experimental) studies have considered the realignment of the Fermi levels of the contacts and the substituted molecules compared to their pristine analogues and the resulting changes in

\footnotetext{
** Tsuji, Y.; Stuyver, T.; Gunasekaran, S.; Venkataraman, L. The Influence of Linkers on Quantum Interference: A Linker Theorem. J. Phys. Chem. C 2017, 121,
} $14451-14462$.

$\Uparrow$ Garner, M. H.; Solomon, G. C.; Strange, M. Tuning Conductance in Aromatic

Molecules: Constructive and Counteractive Substituent Effects. J. Phys. Chem.

C 2016, 120, 9097-9103. 
the measured conductance. $\$, \S \S,{ }^{* * *}$ Proppe and Herrmann reported that bond stretching is capable of increasing the conductance for linear polyenes. ${ }^{. \dagger \dagger}$ Other groups have tried to determine the relationship between the magnitude of conductance under small bias and aromaticity. $+1-\S \S \S$ Some of the authors of the present study connected the magnitude of transmission around the Fermi level $\left(\mathrm{T}_{\mathrm{F}}\right)$ for alternant hydrocarbons to the weight of the diradical resonance structures with radical centers located on the contact positions (see Fig.

\$t Venkataraman, L.; Park, Y. S.; Whalley, A. C.; Nuckolls, C.; Hybertsen, M. S.;

Steigerwald, M. L. Electronics and Chemistry: Varying Single-Molecule Junction Conductance Using Chemical Substituents. Nano Lett. 2007, 7, 502-506.

$\S \S$ Xiao, X.; Nagahara, L. A.; Rawlett, A. M.; Tao, N. Electrochemical Gate-Controlled Conductance of Single Oligo(phenylene Ethynylene)s. J. Am. Chem. Soc. 2005, 127, 9235-9240.

*** Jin, C.; Strange, M.; Markussen, T.; Solomon, G. C.; Thygesen, K. S. Energy Level Alignment and Quantum Conductance of Functionalized Metal-Molecule Junctions: Density Functional Theory Versus GW Calculations. J. Chem. Phys. 2013, 139, 184307.

†† Proppe, J.; Herrmann, C. Communication through Molecular Bridges: Different Bridge Orbital Trends Result in Common Property Trends. J. Comput. Chem. 2015, 36, 201-209.

H. Low, J. Z.; Sanders, S. N.; Campos, L. M. Correlating Structure and Function in Organic Electronics: From Single Molecule Transport to Singlet Fission. Chem. Mater. 2015, 27, 5453-5463.

$\S \S$ Fujii, S.; Marqués-González, S.; Shin, J.-Y.; Shinokubo, H.; Masuda, T.; Nishino, T.; Arasu, N. P.; Vázquez, H.; Kiguchi, M. Highly-Conducting Molecular Circuits Based on Antiaromaticity. Nat. Commun. 2017, 8, 15984. 
1a). ${ }^{* * *}$ In this work, chemical intuition was used to estimate the relative weight of diradical

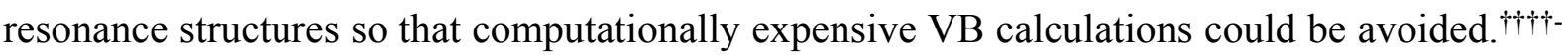

H+t

a)

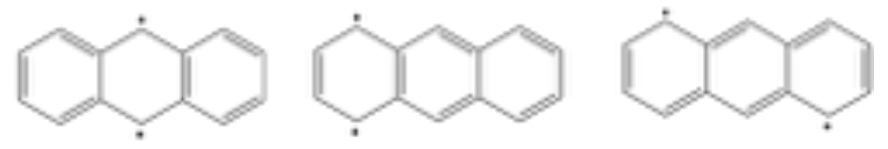

b)

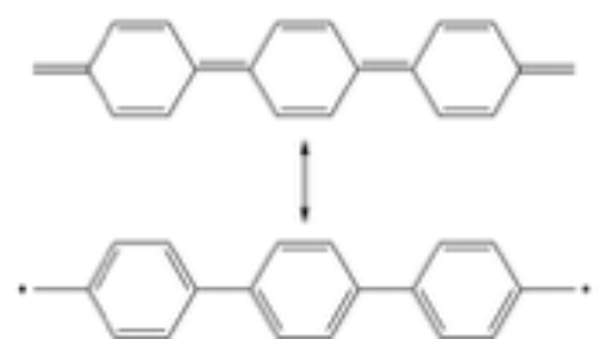

Figure 1. (a) Consideration of the relative weight of specific diradical resonance structures in the resonance hybrid enables an estimation of the magnitude of the transmission probability when contacts are connected to the molecule through the positions corresponding to the radical centers in these structures. The first diradical resonance structure in the figure is relatively stabilized compared to the structures to the right due to the presence of two benzene sextets. ${ }^{16,17}$ The second resonance structure contains a single naphthalene ring and is thus less stabilized. The third structure is not stabilized by aromatic rings. Since the first structure is most stabilized, it has the biggest relative weight in the resonance hybrid and, as a result, the highest transmission probability around the Fermi level will be obtained when contacts are connected to the radical centers in this structure; (b) the quinoid wire (depicted here with three repeating units) predicted to become more conducting with increasing length and its main diradical resonance structure. The weight of this diradical resonance structure in the resonance hybrid increases as more phenyl units are added to the wire, and consequently, the wire will become

\footnotetext{
**** Stuyver, T.; Fias, S.; De Proft, F.; Geerlings, P.; Tsuji, Y.; Hoffmann, R. Enhancing

the Conductivity of Molecular Electronic Devices. J. Chem. Phys. 2017, 146, 092310.

tttt Coulson, C. A. Valence, Clarendon Press, Oxford, 1953.

\$\# Gu, J.; Wu, W.; Danovich, D.; Hoffmann, R.; Tsuji, Y.; Shaik, S. Valence Bond

Theory Reveals the Hidden Delocalized Diradical Character of Polyenes. J. Am.
}

Chem. Soc. 2017, 139, 9302-9316. 
Some of the predictions made in Ref. 16 could be verified through analysis of experimental data found in the literature, for example the increased conductivity of pentacene compared to anthracene when connected to the contacts through the middle rings. $\S_{\S \S}$ Other predictions made in Ref. 16 are harder to assess experimentally, given the expected instability of some of the compounds involved. A remarkable system considered there was a molecular wire which had been proposed before to become more conductive as the wire becomes longer (cf. Fig. 1b), ${ }^{* * * *,+\dagger \dagger \dagger}$ in phenomenological violation of the classical Ohm's law. Unfortunately, an experimental verification of this prediction will be all but impossible, given the known instability of quinoid structures,,$+1+$, $\S \S \S \S$ the building blocks of the proposed wire.

$\S \S \S$ Frisenda, R.; Perrin, M. L.; Valkenier, H.; Hummelen, J. C.; van der Zant, H. S. J. Statistical Analysis of Single-Molecule Breaking Traces. Phys. Status Solidi B 2013, $250,2431-2436$.

***** Ramos-Berdullas, N.; Mandado, M. Electronic Properties of $p$-Xylylene and pPhenylene Chains Subjected to Finite Bias Voltages: A New Highly Conducting Oligophenyl Structure. Chem. Eur. J. 2013, 19, 3646-3654.



Delocalization, Long Bond Order Structure Count and Transmission: An Application to Molecular Wires. Chem. Phys. Lett. 2015, 630, 51.

Hftt Coulson, C. A.; Craig, D. P.; Maccoll, A.; Pullman, A. P-Quinodimethane and its Diradical. Discuss. Faraday Soc., 1947, 2, 36-38.

$\S \S \S \S \S$ Montgomery, L. K.; Huffman, J. C.; Jurczak, E. A.; Grendze, M. P. The Molecular Structures of Thiele's and Chichibabin's Hydrocarbons. J. Am. Chem. Soc. 1986, $108,6004-6011$. 
In this work, we explore a novel way to tune the diradical character of a molecule and ultimately design a wire with the remarkable conductance increasing with the length. ${ }^{* * * * *}$ To this end, we turn to a powerful idea which originated a long time ago in the search for stable radicals and has inspired plenty of applications in different fields ever since: captodative substitution. ${ }^{\dagger \dagger+1 \dagger-1+t+1}$ Such a substitution strategy generally involves an acceptor (capto-) and a donor (-dative). The two components sandwich and stabilize a radical center. The stabilization arises from the energy lowering of the donor's HOMO as it has a bonding interaction with the singly occupied molecular orbital (SOMO) of the radical center; meanwhile the corresponding antibonding interaction is mitigated by the overlap between the SOMO and the acceptor's LUMO (for more information, see Fig. 2 and Ref. 27 and 30). One way to exert the captodative strategy is through $\mathrm{BN}$-substitution, in which $\mathrm{N}$ takes the role of donor and B the role of acceptor (for a list of other potential donor and acceptor components we refer to the work by Pasto and Viehe and co-workers). $. \S \S \S \S, 26$

${ }^{* * * * * *}$ We note here that an increasing conductance of a quantum wire with its length corresponds by no means to a violation of the conservation of current principle; the systems as a whole simply becomes more conducting as more repeating units are added, see also section XI in Ref. 16.

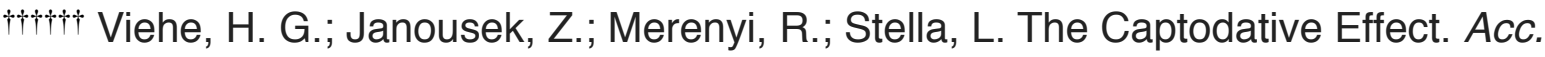

Chem. Res. 1985, 18, 148-154.

\$+1 Mo, Y.; Jiao, H.; Schleyer, P. V. R. Hyperconjugation Effect in Substituted Methyl Boranes: An Orbital Deletion Procedure Analysis. J. Org. Chem. 2004, 69, 3493-3499.

$\S \S \S \S \S \S$ Pasto, D. J. Radical Stabilization Energies of Disubstituted Methyl Radicals. A Detailed Theoretical Analysis of the Captodative Effect. J. Am. Chem. Soc. 1988, 110, 8164-8175. 


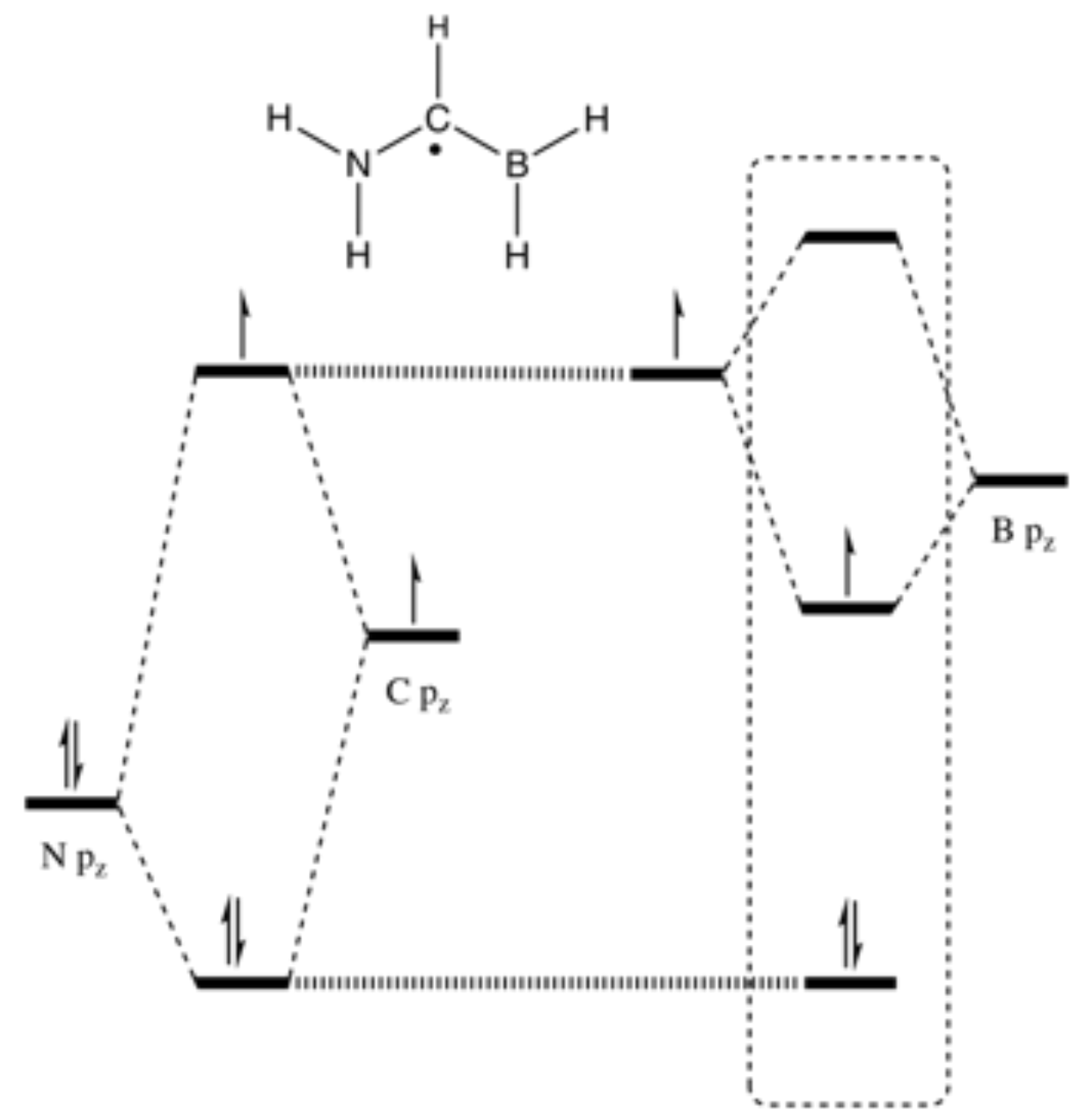

Figure 2. Frontier orbital interaction scheme for captodative stabilization in the $\mathrm{NH}_{2} \mathrm{CHBH}_{2}$ radical. Overall, the orbital interactions stabilize the $\mathrm{BCN}$ system. The doubly-occupied $M O$ in the dashed box is significantly lower in energy than the $\mathrm{N}$ lone pair orbital, more than compensating for the slight destabilization of the singlyoccupied MO in the box compared to the SOMO on C (adapted from Ref. 30). 


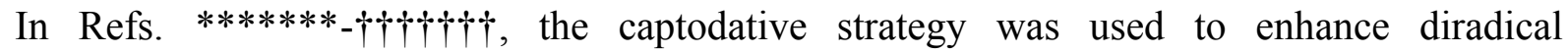

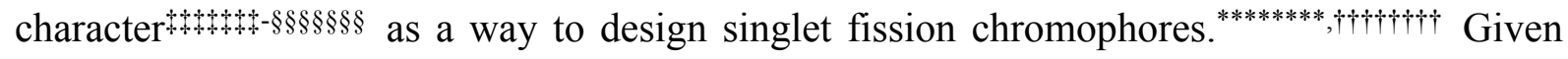
the rationale in Ref. 16, we expect that this strategy can be used to enhance conductance in molecular electronics, also through enhancing diradical character. We report the first attempt in this direction here. Please note the difference between the work of Lissau et al. and ours, although donor-acceptor (DA) substitution is used in both. In the former work, single DA pair is introduced to facilitate the suppression of the effects of cross-conjugation (the presence the QI feature in the transmission spectrum) by environmental influences, i.e., the applied electric field. In the present work, two DA pairs are introduced, each stabilizing one radical center, to enhance the diradical character of the substituted structure, and consequently enhance its conductance directly. The two strategies are fundamentally different.

******* Zeng, T.; Ananth, N.; Hoffmann, R. Seeking Small Molecules for Singlet Fission: A Heteroatom Substitution Strategy. J. Am. Chem. Soc. 2014, 136, 12638-12647. ††††† Akdag, A.; Havlas, Z.; Michl, J. Search for a Small Chromophore with Efficient Singlet Fission: Biradicaloid Heterocycles. J. Am. Chem. Soc. 2012, 134, 14624-14631.

H+tt Yamaguchi, K. Self-Consistent Field: Theory and Applications. by R. Carbo and M. Klobukowski, Elsevier, Amsterdam, 1990, 727.

$\S \S \S \S \S \S$ Minami, T.; Ito, S.; Nakano, M. Fundamental of Diradical-Character-Based

Molecular Design for Singlet Fission. J. Phys. Chem. Lett. 2013, 4, 2133-2137.

Smith, M. B.; Michl, J. Singlet Fission. Chem. Rev. 2010, 110, 6891-6936. ititititi Minami, T.; Nakano, M. Diradical Character View of Singlet Fission. J. Phys. Chem. Lett. 2011, 3, 145-150. 
Another motivation for this work is the great success in azaborine synthesis in the past half a century. The first BN-substituted aromatic compound was reported by Dewartftttt and since then, the knowledge about the synthesis of $\mathrm{BN}$-substituted aromatic hydrocarbons has

increased significantly. $\S \S \S \S \S \S \S \S-* * * * * * * * *$ Recently, BN-substituted fullerenes and their potential use in molecular electronic applications have also started to gain attention. ${ }^{1+1+1+1 \dagger+-1+1+t+t} \mathrm{We}$ believe that the $\mathrm{BN}$-based captodative substitution scheme provides a very promising pathway towards full chemical control of the conductivity of molecules, and cross-links the field of molecular electronics to the likewise vibrant field of azaborine chemistry.

\section{Computational Methods}

$\$+\$+4$ Dewar, M. J. S.; Kubba, V. P.; Pettit, R. New Heteroaromatic Compounds.

Part I. 9-Aza-10-boraphenanthrene J. Chem. Soc. 1958, 624, 3073-3076.

$\$ \$ \S \S \S \S \S$ Jaska, C. A.; Emslie, D. J.; Bosdet, M. J.; Piers, W. E.; Sorensen, T. S.;

Parvez, M. Triphenylene Analogues with B2N2C2 Cores: Synthesis, Structure, Redox

Behavior, and Photophysical Properties. J. Am. Chem. Soc. 2006, 128, 10885-10896.

Campbell, P. G.; Marwitz, A. J. V.; Liu, S.-Y. Recent Advances in Azaborine

Chemistry. Angew. Chem., Int. Ed. 2012, 51, 6074-6092.

ittititi Han, W.; Bando, Y.; Kurashima, K.; Sato, T. Synthesis of Boron Nitride

Nanotubes from Carbon Nanotubes by a Substitution Reaction. Appl. Phys. Lett. 1998, 73, 3085-3087.

tutt Abbaszadeh, B.; Ganji, M. D. Electrical Characteristics of C36 Molecular

Conductor and its B-and N-Doped Isomers. Electron. Mater. Lett. 2013, 9, 63-69. 
The electron transport calculations were performed using the Non-Equilibrium Green's Function (NEGF) method combined with DFT as implemented in the Artaios

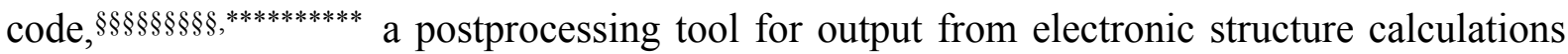
with various quantum chemistry codes, among which Gaussian 09. were chosen as the electrodes and thiolethynyl linkers $(-\mathrm{C} \equiv \mathrm{C}-\mathrm{SH})$ were added to the molecule to facilitate the connection to the contacts. In the first step, the examined structures were

$\S \S \S \S \S \S \S \S \S$ C. Herrmann, G. C. Solomon, J. E. Subotnik, V. Mujica, M. A. Ratner, Ghost Transmission: How Large Basis Sets Can Make Electron Transport Calculations Worse. J. Chem. Phys. 132, 024103 (2010).

Deffner, M.; Groß, L.; Steenbock, T.; Voigt, B. A.; Solomon, G. C.; Herrmann, C. Artaios - a Code for Postprocessing Quantum Chemical Electronic Structure Calculations, available from https://www.chemie.uni-hamburg.de/ac/herrmann/ software/index.html (2008-2017).

tttttttt Frisch, M. J.; Trucks, G. W.; Schlegel, H. B.; Scuseria, G. E.; Robb, M. A.; Cheeseman, J. R.; Scalmani, G.; Barone, V.; Mennucci, B.; Petersson, G. A.; et al. Gaussian 09, Revision D.01, Gaussian, Inc., Wallingford CT, 2009. 
optimized at the B3LYP this optimization, the thiol's hydrogen atoms were removed and Aug clusters, approximating the electrode surface, were attached in accordance with the methodology presented in a recent study. $+4+f+1+\neq$ The adsorption site is the fcc-hollow site. The Au-S distance was set to 2.48 $\AA ̊ . \S \S \S \S \S \S \S \S \S \S \S$ For the resulting structures, single-point calculations were performed at the B3LYP/LanL2DZ level of theory. In the final step, the Hamiltonian and overlap matrices were extracted to carry out the NEGF calculation within the wide-band-limit (WBL)

At+ftt Becke, A. D. Density-Functional Exchange-Energy Approximation with Correct Asymptotic Behavior. Phys. Rev. A 1988, 38, 3098-3100. §§§§§§§§§§ Lee, C.; Yang, W.; Parr, R. G. Development of the Colle-Salvetti CorrelationEnergy Formula into a Functional of the Electron Density. Phys. Rev. B 1988, 37, 785-789.

${ }^{* * * * * * * * * *}$ Becke, A. D. Density-Functional Thermochemistry. III. The Role of Exact Exchange. J. Chem. Phys. 1993, 98, 5648-5652.

tttttttttt Stephens, P. J.; Devlin, F. J.; Chabalowski, C.; Frisch, M. J. Ab Initio Calculation of Vibrational Absorption and Circular Dichroism Spectra Using Density Functional Force Fields. J. Phys. Chem. 1994, 98, 11623-11627.

H+t+t+tł Schlicke, H.; Herrmann, C. Controlling Molecular Conductance: Switching Off $\pi$ Sites through Protonation. ChemPhysChem 2014, 15, 4011-4018. $\S \S \S \S \S \S \S \S \S \S$ Bilić, A.; Reimers, J. R.; Hush, N. S. The Structure, Energetics, and Nature of the Chemical Bonding of Phenylthiol Adsorbed on the Au (111) Surface: Implications for Density-Functional Calculations of Molecular-Electronic Conduction. J. Chem. Phys. 2005, 122, 094708. 
approximation. ${ }^{* * * * * * * * * * * *}$ In the WBL approximation, we used a constant value of $0.036 \mathrm{eV}^{-1}$ for the local density of states (LDOS) of the electrode surface. This value was taken from the literature. ${ }^{63}$ To estimate the diradical character, structures of compounds 1-4 were optimized at the B3LYP/cc-pVTZ level of theory. Complete-active-space self-consistent-field (CASSCF) calculations with the same basis set were carried out for those structures to obtain $n_{L}$ of the ground state wave functions. The active spaces include all orbitals and the lone pair orbitals on $\mathrm{O}$. The monomer calculations were carried out using GAMESS-

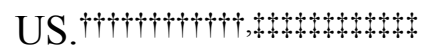

\section{Results and Discussion}

We first take a look at a compound proposed by Zeng et al. ${ }^{30}$ (compound 2 in Fig. 3) as potential candidates for more conducting alternatives for benzene ( 1 in Fig. 3). This $C_{2 h}$ diazadiborine structure has increased diradical character with radical centers in para

${ }^{* * * * * * * * * * * *}$ Herrmann, C.; Solomon, G. C.; Ratner, M. A. Designing Organic Spin

Filters in the Coherent Tunneling Regime. J. Chem. Phys. 2011, 134, 224306. ititititititi Schmidt, M. W.; Baldridge, K. K.; Boatz, J. A.; Elbert, S. T.; Gordon, M. S.; Jensen, J. H.; Koseki, S.; Matsunaga, N.; Nguyen, K. A.; Su, S.; Windus, T. L.; Dupuis, M.; Montgomery, J. A., Jr. General Atomic and Molecular Electronic Structure System. J. Comput. Chem. 1993, 14, 1347-1363.

Gordon, M. S.; Schmidt, M. W., Advances in Electronic Structure Theory: GAMESS a Decade Later in Theory and Applications of Computational Chemistry: The First Forty Years, Dykstra, C. E.; Frenking, G.; Kim, K. S.; Scuseria, G. E. (Eds) 2005, Elsevier, Amsterdam Chap. 41, pp 11167-1189. 
positions; such an electronic structure, according to the rationale laid out in Ref. 16, is expected to increase the conductance compared to the pristine benzene analogue when contacts are connected to the para carbon atoms. The increased diradical character for compound 2 can be intuitively understood as arising from stitching up two of the BNstabilized methyl radicals (cf. Fig. 2). As a reflection of the enhanced diradical character, the occupation number of the lowest unoccupied natural orbitals $n_{L}$, an indicator of the diradical

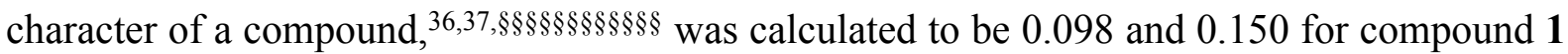
and $\mathbf{2}$, respectively.

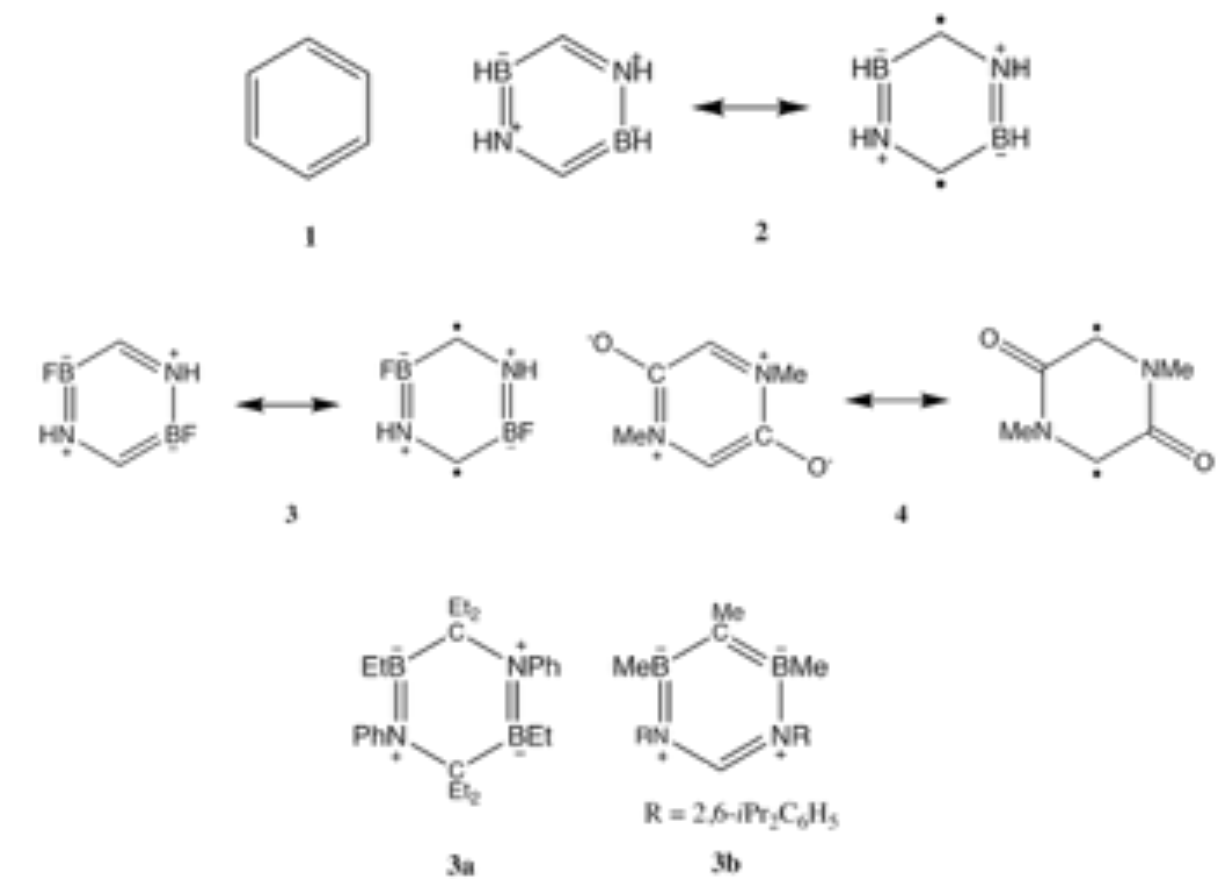

Figure 3. The monomer structures discussed in this manuscript.

$\S \S \S \S \S \S \S \S \S$ Hachmann, J.; Dorando, J. J.; Avilés, M.; Chan, G. K. L. The Radical

Character of the Acenes: A Density Matrix Renormalization Group Study. J. Chem.

Phys. 2007, 127, 134309. 
In order to be able to measure the conductivity of a compound through a Mechanically Controlled Break Junction (MCBJ) or Scanning Probe Microscopy (SPM) experiment, the compound has to be connected to linker units which are responsible for the anchoring of the molecule to the (usually $\mathrm{Au}$ ) contacts. In this work, ethynylthiol linkers were considered (see Fig. 4 for the resulting structures). ${ }^{* * * * * * * * * * * * *}$ Calculation of the transmission spectrum of the resulting structures using the Artaios code ${ }^{55}$ gives the transmission spectra in Fig. 4 (see the Computational Methods section for computational details).

As expected, $\mathrm{T}_{\mathrm{F}}$ of compound $\mathbf{2}$ is much higher than that of compound 1, leading to the conclusion that the conductance under small bias for compound 2 will indeed be enhanced.

Is it possible to enhance the conductivity of benzene even further by modifying substitution patterns? Zeng et $a l .{ }^{30}$ and Akdag et $a l .^{34}$ considered quite a number of captodative substitution schemes, several of which stay within the hexagonal motif of benzene and can be identified as potential stabilizers of diradical resonance structures. We consider two of their most promising substitution schemes that contain even more substantial diradical character (compound $\mathbf{3}$ and 4, cf. Fig. 3). In compound $\mathbf{3}$, the acceptor ability of B is augmented due to the electronegative $\mathrm{F}$ attached to it, leading to a further increase in the diradical character compared to compound $\mathbf{2}\left(n_{l}=0.216\right)$. Compound $\mathbf{4}$, which is -isoelectronic and isosteric to $\mathbf{3}$, has been determined to have an even higher diradical character $\left(n_{L}=0.226\right)$.

\footnotetext{
Sun, L.; Diaz-Fernandez, Y. A.; Gschneidtner, T. A.; Westerlund, F.; Lara-

Avila, S.; Moth-Poulsen, K. Single-Molecule Electronics: From Chemical Design to Functional Devices. Chem. Soc. Rev. 2014, 43, 7378-7411.
} 
From the specific viewpoint of single molecule electronics, compound $\mathbf{4}$ is a very interesting one. It combines two counteracting effects: the transmission probability is enhanced due to an increase in the weight of the diradical character; the diradical resonance structure contains keto groups (cf. Fig. 3), which have been identified as inducers of QI and to block the path of the current. 3 , of these two effects is dominant (will the current be enhanced or decreased around the Fermi level?). The transmission spectra for compounds $\mathbf{3}$ and $\mathbf{4}$ are shown in Fig. 4, in comparison with those of compounds 1 and 2.
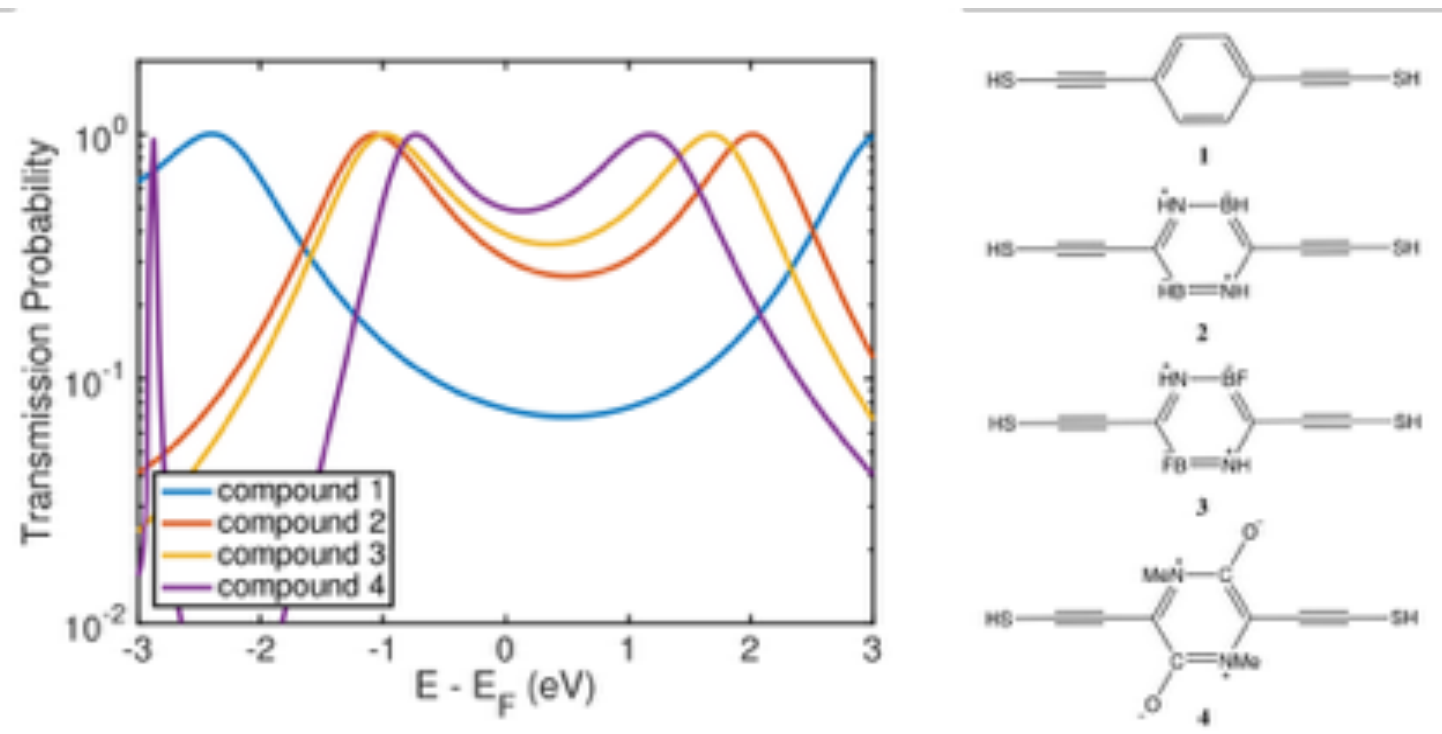

Figure 4. The transmission spectra of compound 1-4.

ititititititi Markussen, T.; Stadler, R.; Thygesen, K. S. The Relation between

Structure and Quantum Interference in Single Molecule Junctions. Nano Lett.

2010, 10, 4260-4265.

t+t+t+t+t+ Strange, M.; Seldenthuis, J. S., Verzijl, C. J. O., Thijssen, J. M.; Solomon, G. C. Interference Enhanced Thermoelectricity in Quinoid Type Structures. J. Chem.

Phys. 2015, 142, 084703. 
It is straightforward to see in Fig. 4 that $\mathrm{T}_{\mathrm{F}}$ increases as one goes from compound $\mathbf{1}$ to compound 4, i.e., in the direction of increasing diradical character. As such, this demonstrates clearly that captodative substitution can indeed be used as a strategy to enhance the conductivity of molecular electronic devices. In the spectrum of compound 4 , no sign of a QI can be observed in the region around the Fermi level, leading to the conclusion that the enhancement effect is dominant. It is worth mentioning that, although neither compounds 3 nor 4 has been synthesized (yet), compounds 3a and 3b (cf. Fig. 3), which share similar

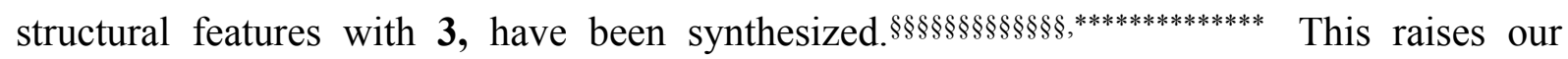
confidence in the realism of the captodative strategy.

Given the substantial increase of conductance in compounds $\mathbf{2 - 4}$, it is now natural to consider employing the captodative strategy to design molecular wires that have their conductance increasing along with their lengths like it was first described for quinoid wires (Fig. 1b). We first compare an oligophenylene wire (wire 1 in Fig. 5) and its BN-substituted analogue (wire 2).

$\S \S \S \S \S \S \S \S \S \S \S \S$ Casanova, J.; Kiefer, H. R.; Kuwada, D.; Boulton, A. H. 1, 3-Diaza-2, 4-

Diboretidines. Isocyanide-Borane Adducts. Tetrahedron Lett. 1965, 6, 703-714. Forster, T. D.; Krahulic, K. E.; Tuononen, H. M.; McDonald, R.; Parvez, M.; Roesler, R. A. A б-Donor with a Planar Six-r-Electron B2N2C2 Framework: Anionic N-Heterocyclic Carbene or Heterocyclic Terphenyl Anion? Angew. Chem., Int. Ed. 2006, 45, 6356-6359. 

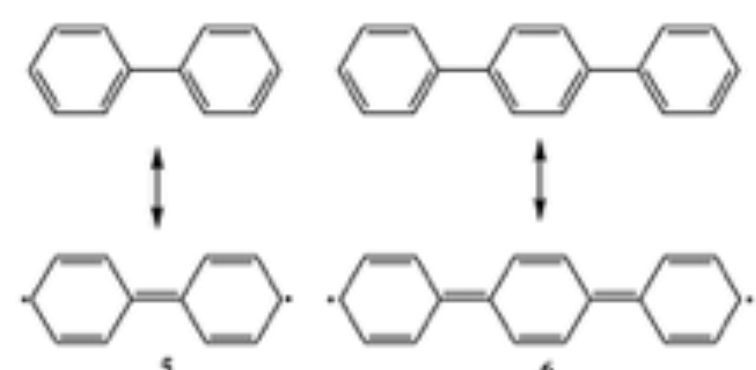

5
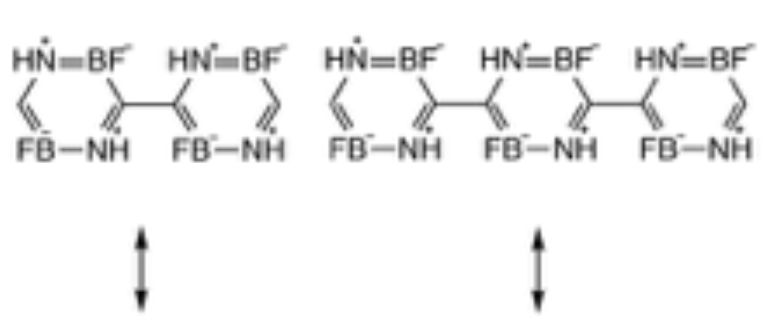

$$
1
$$

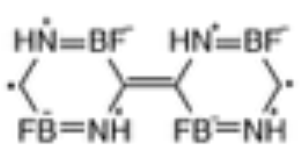

9
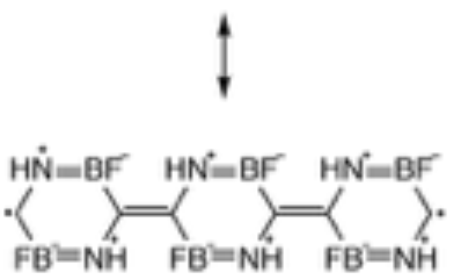

10
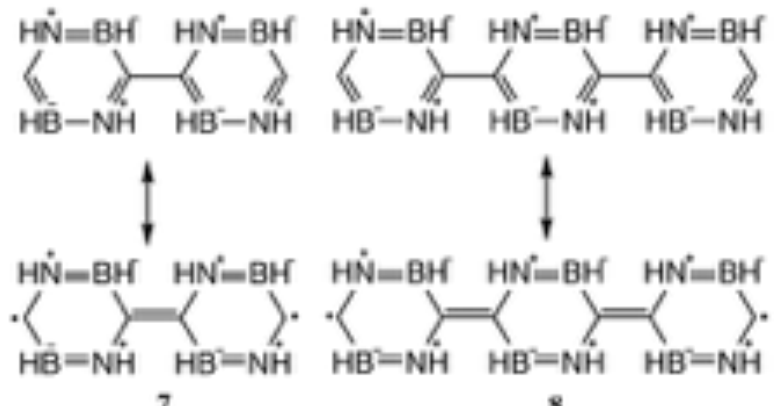

8
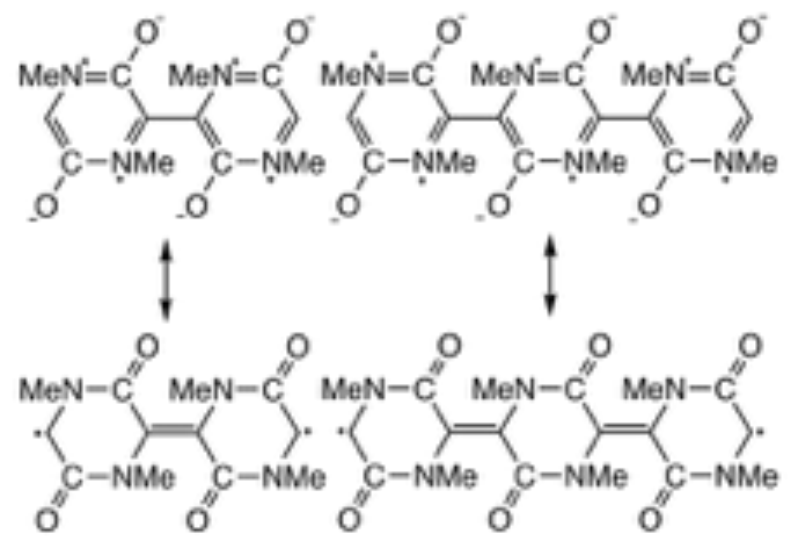

11

12

Figure 5. The wires with compound 1, 2, 3 and 4 as repeating units together with their diradical resonance structures. We will refer to these wires as wires 1, 2, 3 

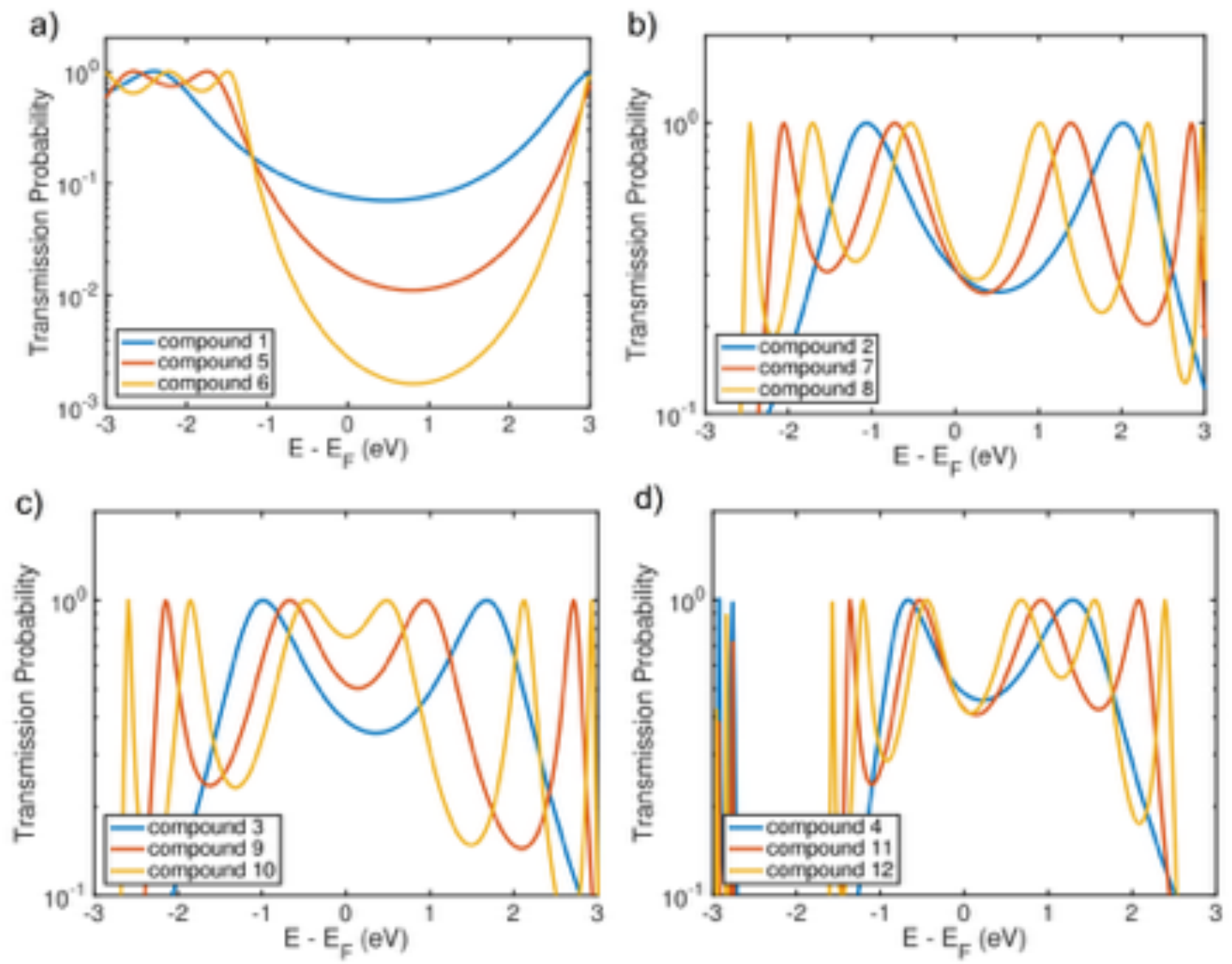

Figure 6. The transmission spectra for (a) wires 1 (compound 1,5,6), (b) 2 (compound 2,7,8), (c) 3 (compound 3,9,10), and (d) 4 (compound 4,11,12).

For the oligophenylene wire, the obtained transmission spectra are presented in Fig. 6a. One clearly observes that, as the length of the wire 1 increases, $T_{F}$ (and thus also the conductance under small bias) decreases. This corresponds to the traditionally expected Ohmic behavior of a molecular wire. The most stable resonance structure of such a wire has all benzene rings in the sextet form. As the length increases, forming the terminal diradical in wire $\mathbf{1}$ requires to break more and more sextet rings and is hence more unfavorable. 
The corresponding transmission spectra of wire 2 are presented in Fig. 6b. Instead of the exponential decrease observed for wire $1, \mathrm{~T}_{\mathrm{F}}$ of wire $\mathbf{2}$ remains largely unchanged as the length of the wire increases and more units are added. The captodative substitution increases the diradical character of the BN-ring and reduces its propensity of having sextet stabilization. When the BN-rings are connected, there will be -covalent coupling between the radical centers on adjacent rings, while the centers at the two ends are still open. This then results in the invariant conductance with increasing length.

The two wires' different behaviors in conductance are consistent with their structures. Wire 2 is entirely flat, although it experiences a similar steric hindrance as the one that causes the dihedral angle between the two phenyl groups in biphenyl. This flatness arises from the formation of inter-ring -bonds and is consistent with an increased weight of the resonance structure with the radical centers at the two ends of the chain. The phenyl units in wire 1, however, have a dihedral angle of $36^{\circ}$ between them and the formation of the inter-ring bonds is frustrated. Each benzene ring is aromatically stabilized; formation of the inter-ring bonds and hence the coplanar configuration is disfavored, and there is no driving force to leave radical centers at the ends. The steric hindrance forces the rings to rotate about the inter-

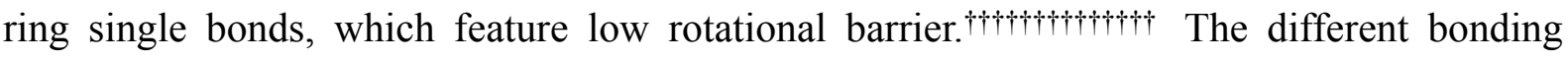
patterns in wires $\mathbf{1}$ and $\mathbf{2}$ are also reflected by the inter-ring $\mathrm{CC}$ bond lengths, which are about 1.48 and $1.44 \AA$, respectively.

thititititi Grein, F. Twist Angles and Rotational Energy Barriers of Biphenyl and Substituted Biphenyls. J. Phys. Chem. A 2002, 106, 3823-3827. 
The invariant conductance of wire 2, although approaching the expected behavior of the quinoid wire in Fig. 1b, is not sufficient. We move on to consider two wires with compounds 3 and 4 as repeating units, respectively (Fig. 5). Fig. 6c demonstrates that $\mathrm{T}_{\mathrm{F}}$ of wire 3 increases significantly as the length of the wire increases; this is exactly the anti-Ohmic conductance that we want to achieve. We note that this wire is perfectly flat as well. In addition to the higher diradical character in $\mathbf{3}$ vs. $\mathbf{2}$, the inter-ring $\mathrm{F} \cdots \mathrm{H}$ hydrogen bonds $(1.98$ $\AA$ ) favor such a coplanar structure and strengthen the inter-ring -bonds. As a reflection of the stronger -bonds, the inter-ring C-C bond lengths in wire $\mathbf{3}$ are even shorter than in wire $\mathbf{2}$ (1.41 $\AA$ vs $1.44 \AA)$.

The transmission spectra for wire $\mathbf{4}$ can be found in Fig. 6d. For this wire, we don't observe the same increase of $\mathrm{T}_{\mathrm{F}}$ with the length as predicted for wire $\mathbf{3}$, even though the diradical character for compound $\mathbf{4}$ is higher (see before). This reversed behavior is caused by the steric hindrance between the methyls and ketones. The dihedral angle between the phenyl units is $52^{\circ}$, which impedes the formation of the inter-ring -bonds and the radical centers at the ends. The decreased conductance of the longer analogues ensues. To verify that this effect can be almost entirely attributed to the steric effect, the evolution of the transmission spectrum with the length for wire $\mathbf{3}$, for which the inter-ring dihedral angles are set to the value of wire $\mathbf{4}$, is presented in Fig. S1 in the Supporting Information. As expected, a drop-off is observed in this case as well, supporting our assertion. Finally, an overview of the behavior of the four wires' $\mathrm{T}_{\mathrm{F}}$ is presented in Fig. S3.

\section{Conclusions}


While it is not entirely clear whether the considered molecular wires are chemically persistent, we think that our captodative substitution strategy to obtain anti-Ohmic nano conductors is definitely worthy of further exploration. More scrutiny of this new substitution strategy to enhance the conductivity of molecules may lead to the proposal of other wires, more feasible to treat experimentally and/or leading to an even more pronounced increase in the conductance with the length of the wire. As such, we think that the present paper not only offers experimental chemists a possible pathway towards verification of the hypothesis outlined, that under small bias molecular wires do not necessarily demonstrate a decreasing conductance as they become longer, but, more generally speaking, provides a proof of principle that captodative substitution is a viable strategy to fine-tune the conductivity of molecules and polymers. The capability to fine-tune the conductivity of molecular electronic devices could be of great interest for the molecular electronics community. For example, this strategy offers a pathway to the design of improved quantum-interference exploiting molecular switches in which the on/off ratio is increased by tuning the conductivity of the onstate (the state without quantum interference) through captodative effects.

\section{Supporting Information}

See Supporting Information for the transmission spectra for wire $\mathbf{3}$ with the dihedral angle between the units set to $52^{\circ}$ and the overview of the transport behavior of the wires with their length.

\section{Acknowledgements}


T.S. acknowledges the Research Foundation-Flanders (FWO) for a position as research assistant (11ZG615N). T.Z. thanks Carleton University of the start-up grant (186853) and the Natural Sciences and Engineering Research Council (NSERC) of Canada for funding (RGPIN-2016-06276). Y.T. thanks Research Institute for Information Technology (Kyushu University) for the computer facilities and financial support from JSPS KAKENHI Grant Number JP17K14440 and from Qdai-jump Research Program, Wakaba Challenge of Kyushu University. S.F. wishes to thank the Research Foundation Flanders (FWO) and the European Union's Horizon 2020 Marie Sklodowska-Curie grant (No 706415)

for financially supporting his postdoctoral research at the ALGC group. P.G. and F.D.P. wish to acknowledge the VUB for a Strategic Research Program. F.D.P. also acknowledges the Francqui foundation for a position as Francqui research professor.

ORCID Thijs Stuyver 0000-0002-8322-0572

ORCID Tao Zeng 0000-0002-1553-7850

ORCID Yuta Tsuji 0000-0003-4224-4532
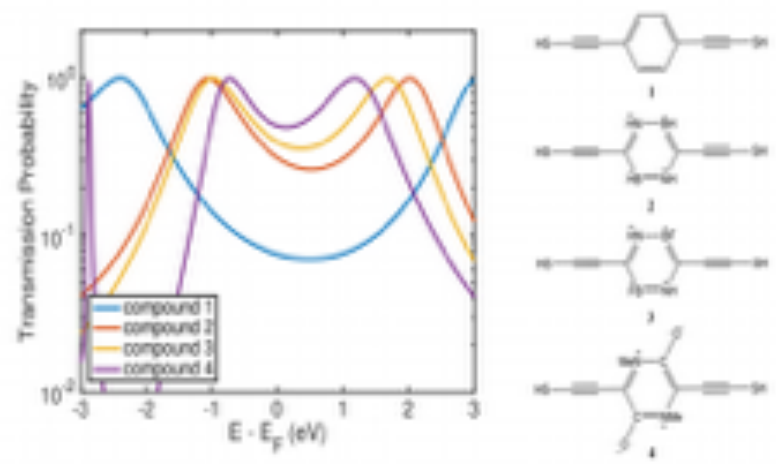

Figure 6. Table of contents graphic. 